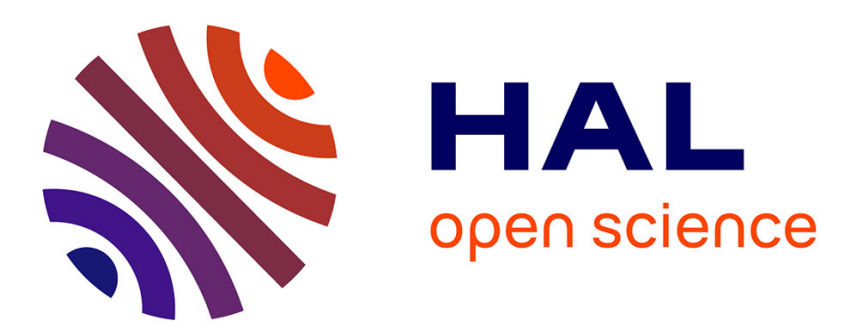

\title{
Three local search meta-heuristics for the Minimum Interference Frequency Assignment Problem (MI-FAP) in cellular networks,
} Yasmine Lahsinat, Dalila Boughaci, Belaid Benhamou

\section{- To cite this version:}

Yasmine Lahsinat, Dalila Boughaci, Belaid Benhamou. Three local search meta-heuristics for the Minimum Interference Frequency Assignment Problem (MI-FAP) in cellular networks,. International Journal of Applied Metaheuristic Computing, 2019, 10 (3), pp.134-150. 10.4018/IJAMC.2019070107 . hal-02098923

\section{HAL Id: hal-02098923 \\ https://hal.science/hal-02098923}

Submitted on 21 May 2020

HAL is a multi-disciplinary open access archive for the deposit and dissemination of scientific research documents, whether they are published or not. The documents may come from teaching and research institutions in France or abroad, or from public or private research centers.
L'archive ouverte pluridisciplinaire HAL, est destinée au dépôt et à la diffusion de documents scientifiques de niveau recherche, publiés ou non, émanant des établissements d'enseignement et de recherche français ou étrangers, des laboratoires publics ou privés. 


\title{
Three Local Search Meta-Heuristics for the Minimum Interference Frequency Assignment Problem (MI-FAP) in Cellular Networks
}

\author{
Yasmine Lahsinat, LRIA-FEI- Computer Science Department, USTHB, BP 32 El-Alia Bab-Ezzouar, 16111, Algiers, Algeria \\ Dalila Boughaci, LRIA-FEI- Computer Science Department, USTHB, BP 32 El-Alia Bab-Ezzouar, 16111, Algiers, Algeria \\ Belaid Benhamou, AIX-Marseille University (AMU), LIS, Domaine Universitaire de Saint Jérôme Avenue Escadrille \\ Normandie Niemen 13397 Marseille Cedex 20, Marseille, France
}

\begin{abstract}
The minimum interference frequency assignment problem (MI-FAP) plays an important role in cellular networks. MI-FAP is the problem of finding an assignment of a small number of frequencies to a large number of transceivers (TRXs) that minimizes the interferences level. The MI-FAP is known to be NP-Hard, thus it cannot be solved in polynomial time. To remedy this, researchers usually use metaheuristic techniques to find an approximate solution in reasonable time. Here, the authors propose three meta-heuristics for the MI-FAP: a variable neighborhood search (VNS) and a stochastic local search (SLS) that are combined to obtain a third and a new one, which is called VNS-SLS. The SLS method is incorporated into the VNS process as a subroutine in order to enhance the solution quality. All three proposed methods are evaluated on some well-known datasets to measure their performance. The empirical experiments show that the proposed method VNS-SLS succeeds in finding good results compared to both VNS and SLS confirming a good balance between intensification and diversification.
\end{abstract}

\section{KEYWORDS}

Assignment Problem, Local Search Methods, Minimum Interference Frequency, Radio Network, Stochastic Local Search (SLS), Variable Neighbourhood Search (VNS), VNS-SLS

\section{INTRODUCTION}

Managing the interferences in a radio network is a relevant issue considering that they can severely damage the quality of the communication. In this paper, we propose to study this issue with a related problem arising in a cellular radio network namely the Minimum Interference Frequency Assignment Problem. The MI-FAP is a well-known challenging combinatorial optimization problem. The issue is to assign a frequency to every transceiver of the network. The difficulties appear due to these two facts: the radio resource is scarce, and the tremendous number of transceivers distributed over the cell's network. Therefore, it is unavoidable to reuse the frequencies multiple times between the transceivers 
within the network. But this will cause interferences. Indeed, when stations in close geographical area use the same or adjacent frequency, interferences occur. To avoid this, it is necessary to assign frequencies to all the geographically close TRXs respecting some required separation constraints.

In a cellular radio network, the objective is then to assign frequencies to TRXs within the different cells in such a way to minimize the total interferences. It is worth recalling these two facts: the TRXs in the same cells cannot use the same frequency and some frequencies are not operational in some cells.

In this work, we consider the two major types of interferences:

- The Co-channel interferences: these interferences appear when two geographically close TRXs use the same frequency (f);

- The Adjacent-channel interferences: these interferences appear if two geographically close TRXs use neighboring (adjacent) frequencies $(f+1, f-1)$.

In the eighties, Hale demonstrated that the considered problem is NP-hard. He proved that the MI-FAP is related to the graph-coloring problem (Hale, 1980). Therefore, the frequency assignment is an important and a complex task when planning a radio network. Considering the limited radio resource, the tremendous number of TRXs, a particular attention is given to achieve this task avoiding or at least minimizing the interferences.

The MI-FAP cannot be solved in polynomial time. Thus, using meta-heuristic techniques offers a good compromise to find approximate solutions for the problem in a reasonable time. Several meta-heuristics have been proposed in the literature. These methods have shown their ability to solve various problems through years. They had been used to handle scheduling problem (Taghavifard, 2012), bioinformatics problem (Zemali, 2016), big data (Benmounah, 2017) and many other problems. In this paper, we propose three meta-heuristic search methods for the MI-FAP. First, we adapt and study the effectiveness of the Variable Neighborhood Search (VNS) for the MI-FAP. Second, we propose a Stochastic Local Search (SLS) method for the MI-FAP. Finally, we propose to merge these two last methods in a new method which we called VNS-SLS. The new method VNS-SLS, includes the SLS method as a local search step to attempt to enhance the solution quality. Since VNS has good diversification properties, integrating SLS in it, will logically result in a good balance between intensification and diversification. We expect then that VNS-SLS will have this feature which is essential for producing good results. We note that collaborative and hybrid methods are worthwhile to ensure a success of optimization tools. There are tremendous works that used hybrid approaches to handle optimization problems. Among them, we mention some recent works (Wang, 2017), (Boucheham, 2017), (Abunaser, 2015).

The rest of this paper is organized as follows: section 2 gives a background on the considered problem. In section 3, we propose a brief literature review. Section 4 explains the key components of the three proposed methods for solving the MI-FAP. Section 5 presents some results of various tests and experimentations. Finally, section 6 concludes the work and gives some future perspectives.

\section{BACKGROUND}

We present in this section the formal description of the MI-FAP used in this paper. Let us consider a radio network $N$ composed of a set of cells: $C=\left\{C_{1}, C_{2} \ldots, C_{i}, \ldots, C_{n c}\right\}$, where $\boldsymbol{n c}$ is the number of cells. Each cell $C_{i}$ having $x_{\mathrm{i}}$ TRXs requires $x_{i}$ different frequencies. The networks manage a fixed number $N F$ of frequencies. Indeed, each network has its own interval $\left[F_{\text {min }}, F_{\text {max }}\right]$ of operational frequencies with $\left|F_{\min }-F_{\max }\right|=N F$. For each cell, we have a set of operational frequencies. This means that some cells could suffer from some eventual restrictions and cannot use some of the frequencies. The cochannel and adjacent channel constraints are represented by two matrices denoted $\mathrm{IM}_{\text {co }}$ respectively $\mathrm{IM}_{\mathrm{adj}}$ that are defined as follows: 
- $\quad \mathbf{I M}_{\mathbf{c o}}[\mathbf{n c}][\mathbf{n c}]$ : Contains the co-channel rate between each pair of cells of the network. An element $I M_{c o}[i][j]$ of the $\mathrm{IM}_{\mathrm{co}}$ matrix corresponds to the rate of co-channel interference when a TRX of the cell $j$ uses the same channel $(f)$ than that one of a TRX $t$ of the cell $I$;

- $\quad \mathbf{I M}_{\text {adj }}[\mathbf{n c}][\mathbf{n c}]$ : Contains the adjacent-channel rate between each pair of cells of the network. An element $I M_{a d j}[i][j]$ of the $\mathrm{IM}_{\text {adj }}$ matrix corresponds to the rate of the Adjacent-channel interference when a TRX of the cell $j$ uses an adjacent channel to a TRX $t$ of the cell $i$.

These two matrices IM $\mathrm{I}_{\text {co }}$ and $\mathrm{IM}_{\text {adj }}$ contain the interaction between cells when the TRXs use the same or adjacent channel. The interferences are expressed as rates (their values are between 0 and 1).

We note that, area-based and traffic-based are two main ways to rate interferences. In this paper, we consider the area-based way where the interference describes the affected area and an element $I M_{c o}[i][j]$ (or $I M_{a d j}[i][j]$ ) describes the percentage of pixels in the service area (Kuurne, 2001) of cell $i$ affected by the cell $j$.

The MI-FAP handles $d$ variables where each variable corresponds to a frequency assigned to a TRX $i$ within a cell $j$. The value of a frequency is picked from the range 1 to $N F$.

The MI-FAP is an optimization problem that consists in finding a frequency assignment for the $d$ TRXs of the network that minimizes the cost function proposed in (Kuurne, 2001) and given in the following formula:

$$
\sum_{i=0}^{i=n c j=n c} \sum_{j=0}\left(Y_{i j} \times I M_{c o}[i][j]+Z_{i j} \times I M_{a d j}[i][j]\right) \times W_{i}
$$

The objective function (1) minimizes the overall interferences that equal to the total co-channel and adjacent channel interference, taking into account the traffic over a cell:

- $W_{i}$ : is a weight associated to the Cell $i$ due to the amount of traffic carried on it;

- $Y_{i j}$ : is the number of different pairs of transceivers of the cell $C_{i}$ and $C_{j}$ that operates on the same frequency;

- $\quad Z_{i j}$ : is the number of different pairs of transceivers of the cell $C_{i}$ and $C_{j}$ that operates on an adjacent frequency $(f),(f+1 / f-1)$.

\section{RELATED WORKS}

The frequency assignment problem (FAP) has a practical interest in both real and theoretical aspects. As the quality of the service of a wireless system depends highly on the optimal management of the radio resource, the FAP is a core problem in the modern wireless systems. It has relevance in many fields such as military and civil applications. The FAP is a challenging combinatorial optimization problem that deals with the tuning of a tremendous number of transceivers, their positions, their frequencies and the interferences that could occur.

The pioneer who introduced the frequency assignment problem is Metzger. He published his research in this field in the sixties (Metzger, 1970). Since that, many researchers investigated this problem (Aardal, 2007; Eisenblatter, 2002; Leese, 2002). Several formulations of the FAP had been proposed. The survey published in 2007 by (Aardal, 2007) summarized the different formulations and objectives associated with the FAP. He said that in general, the objective associated with the FAP is the minimization of the number of frequencies, the range of the spectrum (span), or the total interferences. Therefore, depending on the objective function to optimize, the FAP can be classified as follows (Aardal, 2007): 
- The Minimum Order Frequency Assignment (MO-FAP) attempt to minimize the number of the frequencies used;

- The Minimum Span Frequency Assignment (MS-FAP) attempt to reduce the interval of frequencies and get as small as possible the difference between the highest and the lowest frequency. Most of the early works were devoted to this goal (Mabed, 2011);

- The Minimum Blocking Frequency Assignment (MB-FAP) attempt to assign frequencies in such a way that no unacceptable interference occurs and the overall blocking probability of the network is minimized. More formally the problem is defined (Eisenblätter, 2000);

- The Minimum Interference Assignment Problem (MI-FAP) consists in minimizing the overall interferences.

Regardless of its formulation, the FAP rapidly grew in popularity and attracted many researchers over the last fifty years. We focus in this work on the MI-FAP variant. The MI-FAP is an interesting problem of operational research arising in radio networks where the issue is to assign frequencies to each TRX with the minimum level of interferences. Hale (Hale, 1980) modeled the problem as a graph coloring problem and demonstrated that the considered problem is an NP-hard.

Several works proposed the meta-heuristic framework to handle different datasets of the MI-FAP. Among them the ones that adopted evolutionary approaches such as EAs and Ant Colony optimization algorithms (ACO/EAs) proposed in 2007 (Luna, 2007), or the adaptation of the cultural algorithm (Alami, 2008) published in 2008. Another research studied the particle swarm to handle the FAP (Bezuidenhout, 2014). A recent work addressed the MI-FAP with a Harmony Search (Lahsinat, 2017). Further, there are works that adopted some single based solution meta-heuristic methods such as the tabu search algorithm to solve the problem (Dorne, 1999), (Montemanni R. M., 2003), (Montemanni, 2010), (Lai, 2015). Some other works that dealt with the problem by using the simulated annealing principle (Duque-Anton, 1993), (Beckmann, 1999), (Colombo, 2010). Other works adopted hybrid methods (Luna, 2011), (Mabed, 2011) to solve the problem.

\section{THE PROPOSED APPROACHES}

In this section, we present the main elements of the proposed approaches. We first describe the shared elements: the solution encoding, the objective function measuring the quality of the solutions and the methodology adopted to update the solutions manipulated by all the techniques. Then we describe in detail the three proposed methods SLS, VNS and VNS-SLS.

\subsection{The solution Encoding}

An important step when designing a meta-heuristic is the solution encoding. Indeed, encoding rightly a solution can influence the performances of the algorithms. We consider as a solution to the MIFAP a frequency plan that minimizes the interferences. A solution is expressed by a vector $S o l$ of $d$ elements with $d$ the total number of TRXs. Each element from Sol represents the frequency assigned to the $K^{\text {th }}$ TRX of a cell $i$. Each frequency is represented by a number from 1 to NF:

Sol $\leftarrow\{\mathrm{f} 1 ; \mathrm{f} 2 \ldots \mathrm{fd}\}$

\subsection{Updating Strategy}

Typically, for the MI-FAP, the updating scheme consists in changing the frequency assigned to the TRXs. The strategy adopted must consider these two important elementary conditions: do not assign the same frequency to TRXs within the same cell and do not assign a non-operational frequency. Thus, we adopt an updating strategy that guarantees before performing any changes of frequency 
that the new frequencies are valid and the required separation between the assigned frequencies to TRXs in the same cell is respected.

\subsection{The Objective Function}

The purpose here is to reach a solution that would minimize the level of interferences. In other words, we must find an assignment of the frequency that minimizes the objective function encoded by the formula already given in Equation (1).

\subsection{The Variable Neighborhood Search for MI-FAP}

The variable Neighborhood Search (VNS) algorithm is a single solution oriented meta-heuristic proposed in 1997 by Mladenovic and Hansen (Mladenovic, 1997). Since then various variants of VNS were proposed, but the fundamental idea is the systematic change of neighborhood combined with a local search. This method confirmed its performance to solve several optimization problems (Mladenovic, 1997).

In order to use VNS algorithm, we must define some elements:

1. A solution that the VNS manipulates;

2. A neighborhood structure that should be defined as $N_{k}\left(k=1 \ldots k=k_{\max }\right)$;

3. A local search routine;

4. A stopping condition, which could be the maximum number of iterations or the maximum CPU time.

In the specific case of MI-FAP, the elements of the VNS are defining as follows:

- A Solution: Corresponds to a frequency plan;

- A Neighbor solution: Is a solution generated by a move from the initial solution. The move is the operator that modifies the assignment of one or more TRXs in the initial solution, depending on the value of the $k_{\max }$;

- A Local Search: We adopted a basic one. It starts with an initial solution. In this local search, we choose to generate the neighbor by modifying the conflicting element: we find the frequency assigned to a TRX, that causes the most interferences with its neighbors and we change its frequency to the less used frequency. Then we try to improve it until a stopping criterion is met.

The variable neighborhood search (VNS) process consists in three main phases:

- Initialization phase: Defines the set of neighborhood structure $N_{k}$ and generates the initial solution $x$;

- Main phase: (1) Set $k:=1$. (2) Until $k:=k_{\max }$ repeat the following steps: a) A shaking step: in this step, the process randomly generates $x^{\prime}$, with $\begin{gathered}x \\ x^{\prime} \in N_{k}\end{gathered}$. b) A local search step: in this step the process applies a local search on the obtained neighbor $\mathrm{x}$ ' to obtain a locally optimal solution $x^{\prime \prime}$;

- Acceptance phase: Based on the solution quality, decides either to accept the obtained solution or move to the next neighborhood structure. Formally:

- If $x^{\prime \prime}$ is better than the current solution $x$ set $x:=x^{\prime \prime}$ and restart the process from the first neighborhood structure $N_{1}$ with x" as an initial solution;

- Otherwise set $k:=k+1$. 
For the considered problem, a solution corresponds to a frequency plan. The initial solution is generated randomly. A neighbor solution $x^{\prime}$ of the solution $x$, is defined as a solution with $k$ decision variables having different values comparing to $x$. For the MI-FAP, this means that between the solution $x$ and its neighbor $x^{\prime}$ we have $k$ transceivers with different frequencies. The neighboring solution is obtained by applying a move from the initial solution. This move is an operator that modifies the assignment of frequencies to one or more TRXs in the initial solution $x$. The number of frequencies to modify in the solution $x$ depends on the current value of $N_{k}$. The value of $k_{\max }$ corresponds to the maximum number of different frequencies assigned to TRXs between the solution $x$ and its neighbor $x^{\prime}$.

The pseudo code of VNS is given in Algorithm 1.

\subsection{The Stochastic Local Search for MI-FAP}

The Stochastic local search methods are efficient search techniques to tackle a large range of combinatorial problems. They covered a large class of algorithms. We distinguish between simple iterative improvement methods and complex SLS methods, such as Ant Colony Optimization and Evolutionary Algorithms (Holger H. Hoos, 2004). Hoos and Stutzle mention that many highperformance SLS algorithms are based on a combination of several simple search strategies, such as Iterative Best Improvement and Random Walk. The considered SLS used in this paper probabilistically makes a choice between a random walk and Min-Conflicts inspired from the one developed to handle the optimal winner determination problem (WDP) in combinatorial auctions published in (Boughaci, 2008).

The proposed SLS technique for the MI-FAP starts with an initial solution $x$ and tries to find a better solution in the current neighborhood. The neighboring solution $x^{\prime}$ of the solution $x$ is obtained by modifying the frequency assigned to a selected TRX. The perturbed TRX is selected according to one of the two following criteria:

\section{Algorithm 1. The VNS for the MI-FAP}

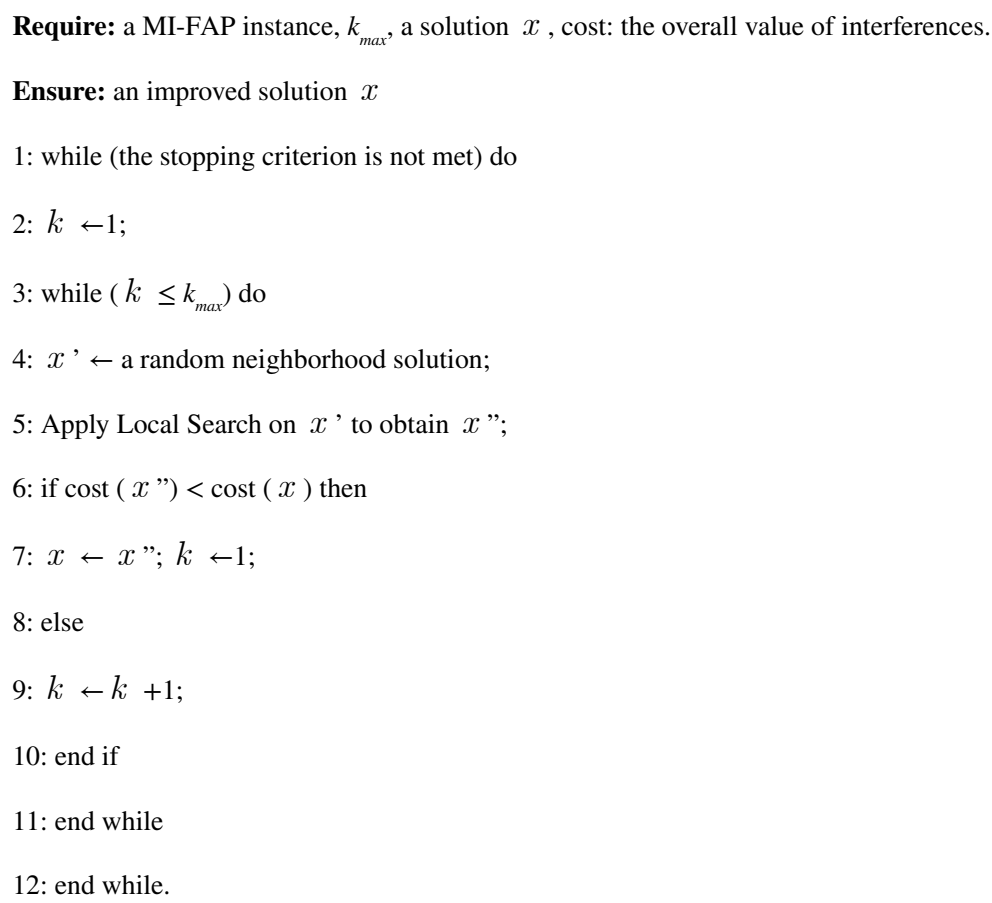


- The first criterion consists in choosing the TRX in a random way with a fixed probability wp $>0$;

- The second criterion consists in choosing the conflicting node: we find the assignment that causes the most interference with its neighbors and then we make a move on it by assigning to the corresponding TRX the less used frequency. The process is repeated until we met stopping criterion.

The pseudo code of SLS for MI-FAP is given in Algorithm 2.

\subsection{A New Local Search Method VNS-SLS for MI-FAP}

In this section, we present the main components of the proposed new approach VNS-SLS that combines both the Variable Neighborhood Search (VNS) and the Stochastic Local Search (SLS) methods. The VNS-SLS is an iterative procedure, which starts from an initial solution $x$ (i.e a frequency assignment plan) generated randomly, and moves step by step in the search space with the aim to improve the initial solution. At each iteration, a move to a neighbor of $x$ is performed. The move consists in changing the assignment of frequency to a TRX selected in the neighborhood of $x$. The path between the initial solution $x$ and the generated neighbor is fixed with the $k_{\max }$.

The SLS step in the VNS-SLS method is a local search process that is used to improve the current solution. This SLS process uses a probability to control intensification and diversification. We integrated this in VNS because Stochastic local search (SLS) algorithms are among the most prominent and successful techniques for solving several computationally difficult problems in many areas of computer science and operations research, including propositional satisfiability, constraint satisfaction, routing, and scheduling (Hoos, 2004).

More precisely, in this work, we adopted a simple SLS as described in section 4.5. It allows two choices: (1) a random walk and (2) a min-conflict algorithm using a probability parameter. These two choices make a balance between intensification and diversification. Indeed, the pure min-conflict algorithm alone will lead to a local optimum. Thus, to avoid being stuck in a local optimum, we introduce a noise through a random walk. It takes the generated neighbor of $x$ and tries to improve it.

\section{Algorithm 2. The SLS for MI-FAP}

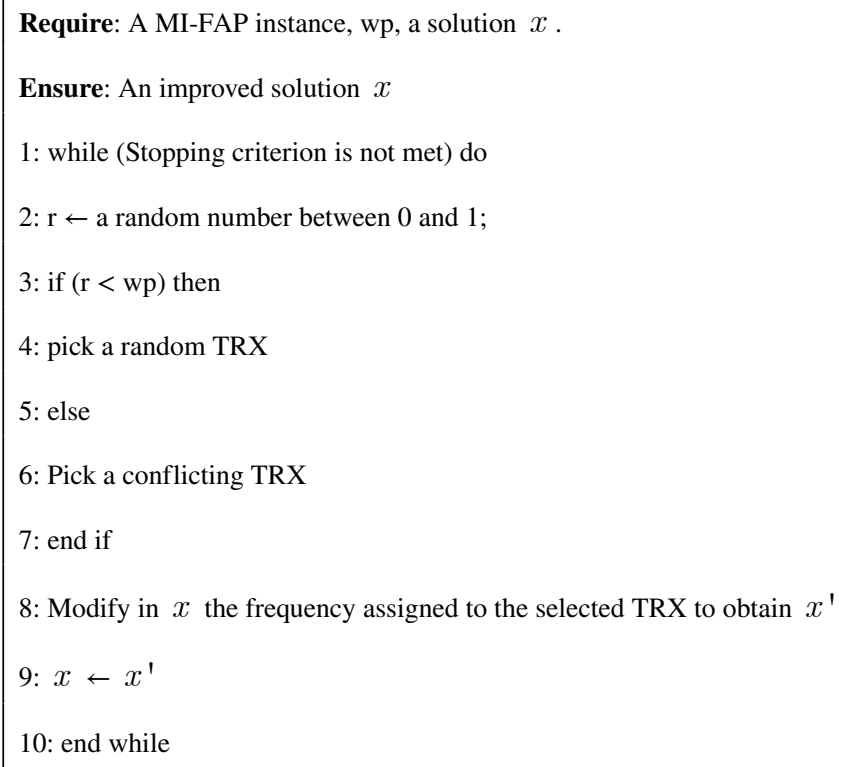


The solution $x$ " obtained at the end of the SLS process will be the current solution in the VNS process when $x^{\prime \prime}$ is better than $x$. Otherwise, the process restarts with the new neighborhood. The process is repeated until a stopping criterion is met.

The VNS-SLS method for MI-FAP is sketched in Algorithm 3.

\section{COMPUTATIONAL EXPERIMENTS}

In this section, we present the computational experiments achieved to check out the performances of the three methods. The experiments are carried on PC having an Intel Core i5 with 4GB of RAM under Windows 7 . We developed the methods using the Java language programming.

\subsection{The Considered Datasets}

We evaluated our methods on several public instances. Due to the non-deterministic nature of the three methods VNS, SLS and VNS-SLS, we consider 10 runs by instance and by algorithm. All instances were picked up from the cost 259 Benchmarks that are available on (Eisenblätter, 2000). We evaluated VNS, SLS, and VNS-SLS on seven different instances of the considered problem namely: Tiny, K, Swisscom, Siemens1, Siemens2, Siemens3, and Siemens4. The COST means European Union Forum for cooperative scientific research (Eisenblatter, 2002). The COST 259 contains several realistic radio network planning scenarios.

We give some details on the instances tackled (Eisenblätter, 2000):

Siemens 01: Contains 506 cells, 930 TRXs, 74 different frequencies available with a total number of 20417 Co-channel interferences and 10344 adjacent-channel interferences to be avoided.

\section{Algorithm 3. The VNS-SLS for MI-FAP}

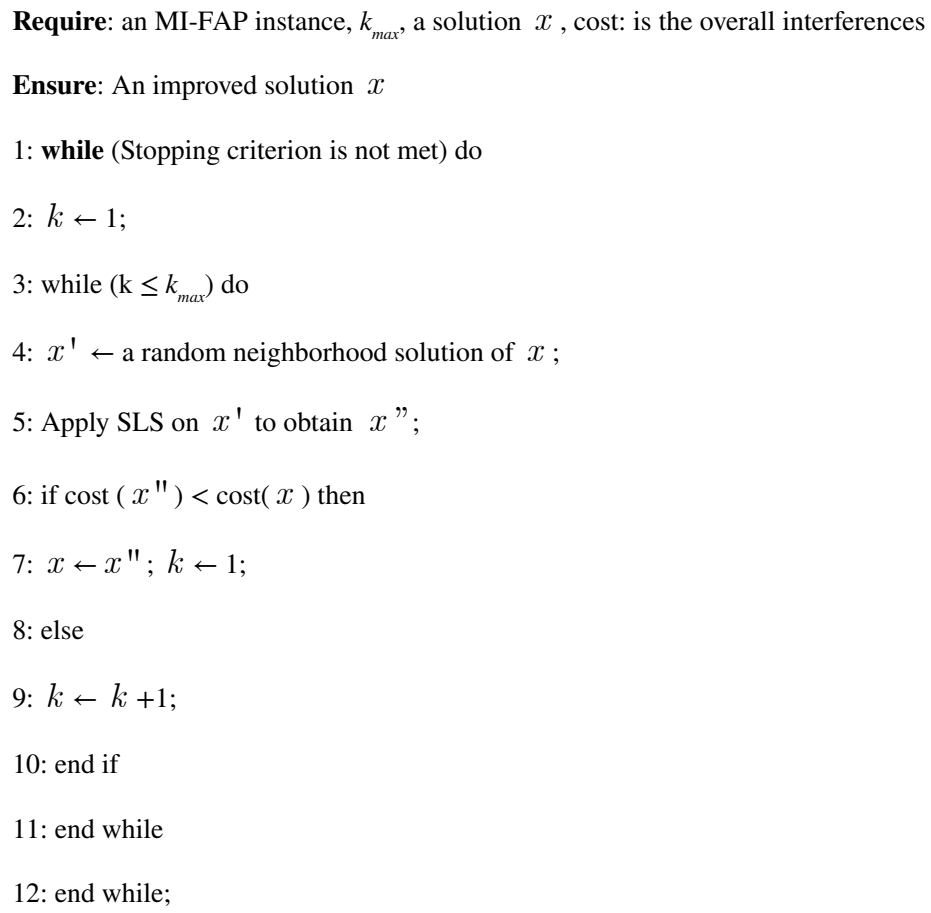


Siemens 02: Includes 254 cells, 977 TRXs, 82 different available frequencies with a total number of 30982 Co-channel interferences and 13970 adjacent-channel interferences.

Siemens 03: Contains 894 cells, 1623 TRXs, 55 different available frequencies with a total number of 63893 Co-channel interferences and 25105 adjacent channel interferences.

Siemens 04: Has 760 cells, 2785 TRXs, 38 different available frequencies, 113658 Co-channel interferences, and 64341 adjacent channel interferences to be avoided.

Swisscom: Composed by 148 cells, 310 TRXs, 47 available frequencies and a total number of 535 adjacent channel interferences.

K: 264 cells, 267 TRXs, 49 frequencies, 27123 Co-channel interferences, and 3199 adjacent channel interferences to be avoided.

Tiny: 7 cells, 12 TRXs, 12 frequencies, 12 Co-channel interferences constraints, and 9 adjacent channel interferences to be avoided.

\subsection{The Parameter Tuning}

For a fair comparison among the three algorithms, we run all the techniques using the same CPU time (1800 seconds). The adjustment of the different parameters of the proposed approaches is fixed by an empirical study:

- The VNS parameters: $k_{\max }=4$, CPU time for the local search sub-routine (LS) $=10$ seconds;

- The SLS parameters: $\mathrm{wp}=0.25$;

- The VNS-SLS parameters: $k_{\max }=4$, wp $=0.25$, CPU time for SLS $=10$ seconds.

\subsection{The Effect of $k_{\max }$}

In order to propose an efficient Variable Neighborhood Search algorithm, we dedicate our first sets of experiments to find the adequate value of the $k_{\max }$ parameter which is very important in the VNS algorithm. We examined five possible values of $k_{\max }=2,3,4,5,6$, and achieve some experiments by using two instances as shown in Table 1 .

The results presented in Table 1 indicate that the best solutions are found when using a $k_{\max }=4$. It is obvious that increasing the size of the neighborhood has a positive effect on the performances. Indeed, it brings enough diversity that avoids being stuck in a local optimum. However, if the size is too large it affects negatively the quality of the solution.

\subsection{The Obtained Results}

Table 2 gives the numerical results of all of the VNS, SLS and VNS-SLS methods on various instances of the MI-FAP problem. The mean, the best, the worst cost value and the Standard deviation (Std) results found by each method are given. The mean value corresponds to the solution quality found by each algorithm on 10 runs. The best and the worst values are respectively the best and worst solutions obtained by each algorithm on 10 runs. The best results, in term of mean and best value, are in bold font.

The results from Table 2 show that VNS-SLS and SLS methods succeed in finding good results for almost the considered instances. They outperform the VNS algorithm for the Siemens and K

Table 1. Results obtained over two instances with different values of $k_{\max }$

\begin{tabular}{|l|l|l|l|l|l|}
\hline Instances & VNS-1 $\left(\boldsymbol{k}_{\max }=\mathbf{2}\right)$ & $\mathbf{V N S - 2}\left(\boldsymbol{k}_{\max }=\mathbf{3}\right)$ & $\mathbf{V N S - 3}\left(\boldsymbol{k}_{\max }=\mathbf{4}\right)$ & $\mathbf{V N S - 4}\left(\boldsymbol{k}_{\max }=\mathbf{5}\right)$ & VNS-5 $\left(\boldsymbol{k}_{\max }=\mathbf{6}\right)$ \\
\hline Siemens01 & 89.71 & 88.46 & 82.18 & 88.8 & 90 \\
\hline K & 98.23 & 97.58 & 88.06 & 91.7 & 98.5 \\
\hline
\end{tabular}


Table 2. The results obtained by the three proposed methods

\begin{tabular}{|c|c|c|c|c|}
\hline \multirow{2}{*}{ Instances } & \multirow{2}{*}{ Statistical Features } & \multicolumn{3}{|c|}{ Method } \\
\hline & & VNS-SLS & SLS & VNS \\
\hline Siemens 01 & $\begin{array}{l}\text { Best } \\
\text { Mean } \\
\text { Worst } \\
\text { std }\end{array}$ & $\begin{array}{l}3.72 \\
4.49 \\
4.90 \\
1.06\end{array}$ & $\begin{array}{l}4.42 \\
6.62 \\
12.21 \\
2.54\end{array}$ & $\begin{array}{l}65.31 \\
82.18 \\
102.94 \\
12.08\end{array}$ \\
\hline Siemens 02 & $\begin{array}{l}\text { Best } \\
\text { Mean } \\
\text { Worst } \\
\text { Std }\end{array}$ & $\begin{array}{l}2567.22 \\
4988.30 \\
8476.13 \\
2774.46\end{array}$ & $\begin{array}{l}2663.20 \\
\underline{3000.81} \\
4309.33 \\
470.77\end{array}$ & $\begin{array}{l}3681.11 \\
5245.68 \\
5643.34 \\
525.57\end{array}$ \\
\hline Siemens 03 & $\begin{array}{l}\text { Best } \\
\text { Mean } \\
\text { Worst } \\
\text { Std }\end{array}$ & $\begin{array}{l}435.11 \\
\underline{506.88} \\
613.98 \\
56.77\end{array}$ & $\begin{array}{l}772.48 \\
963.36 \\
1189.63 \\
120.78\end{array}$ & $\begin{array}{l}970.49 \\
1281.83 \\
1939.33 \\
420.59\end{array}$ \\
\hline Siemens 04 & $\begin{array}{l}\text { Best } \\
\text { Mean } \\
\text { Worst } \\
\text { Std }\end{array}$ & $\begin{array}{l}58204.47 \\
\underline{62816.43} \\
68462.09 \\
3481.577\end{array}$ & $\begin{array}{l}62820.30 \\
67183.12 \\
72804.13 \\
2625.90\end{array}$ & $\begin{array}{l}88035.89 \\
91042.84 \\
93194.87 \\
1638.42\end{array}$ \\
\hline Tiny & $\begin{array}{l}\text { Best } \\
\text { Mean } \\
\text { Worst } \\
\text { Std }\end{array}$ & $\begin{array}{l}0 \\
0.14 \\
1.46 \\
0.46\end{array}$ & $\begin{array}{l}0 \\
\underline{0.08} \\
0.48 \\
0.15\end{array}$ & $\begin{array}{l}0 \\
0.08 \\
0.48 \\
0.18\end{array}$ \\
\hline Swisscom & $\begin{array}{l}\text { Best } \\
\text { Mean } \\
\text { Worst } \\
\text { Std }\end{array}$ & $\begin{array}{l}0 \\
0 \\
0 \\
0\end{array}$ & $\begin{array}{l}0 \\
0 \\
0 \\
0\end{array}$ & $\begin{array}{l}0 \\
0.34 \\
1.33 \\
0.46\end{array}$ \\
\hline K & $\begin{array}{l}\text { Best } \\
\text { Mean } \\
\text { Worst } \\
\text { std }\end{array}$ & $\begin{array}{l}12.31 \\
15.93 \\
18.63 \\
2.21\end{array}$ & $\begin{array}{l}11.30 \\
13.58 \\
16.56 \\
1.82\end{array}$ & $\begin{array}{l}35.59 \\
88.06 \\
265.62 \\
90.47\end{array}$ \\
\hline
\end{tabular}

instances. We can see that the three methods are comparable on the Tiny and Swisscom instances. This is due to the nature of these two problems which are small instances.

For the Tiny instance, we observe that the three methods find the optimal solution (i.e the best $=0$ ). Both VNS-SLS and SLS find the optimal solution for the 10 performed runs on the Swisscom instance.

For the K instance, we observe that SLS and VNS-SLS are comparable with a slight performance in favor of the SLS method. For this instance, we depict in Figure 1 the evolution of the cost value over the 30 minutes of the experiment for the three methods. The curve shows that VNS-SLS is faster than VNS. We can see that for the same time, the result achieved by VNS-SLS is better than the standard VNS with a local search. This means that the combination of SLS within VNS enhances the performances of VNS in term of both the time and the quality point of view. The SLS allows escaping from some bad regions which leads to better results.

For the difficult instances, the results obtained by VNS-SLS and SLS are comparable. However, for the Siemens 1, Siemens 3 and Siemens 4 instances, we can see a slight performance in favor of VNS-SLS. The standard deviation is relatively small. In order to show clearly the performance of the proposed approaches in solving MI-FAP, we draw the bar plots in Figure 2. This Figure compares the three methods VNS, VNS-SLS and SLS in term of quality of solution on the Siemens and K instances. We can see that, the two methods SLS and VNS-SLS explore the neighbor search efficiently. This allows locating good quality solution comparing to the VNS. When using the SLS 
Figure 1. The cost evolution over 30 minutes for the $\mathrm{K}$ instance

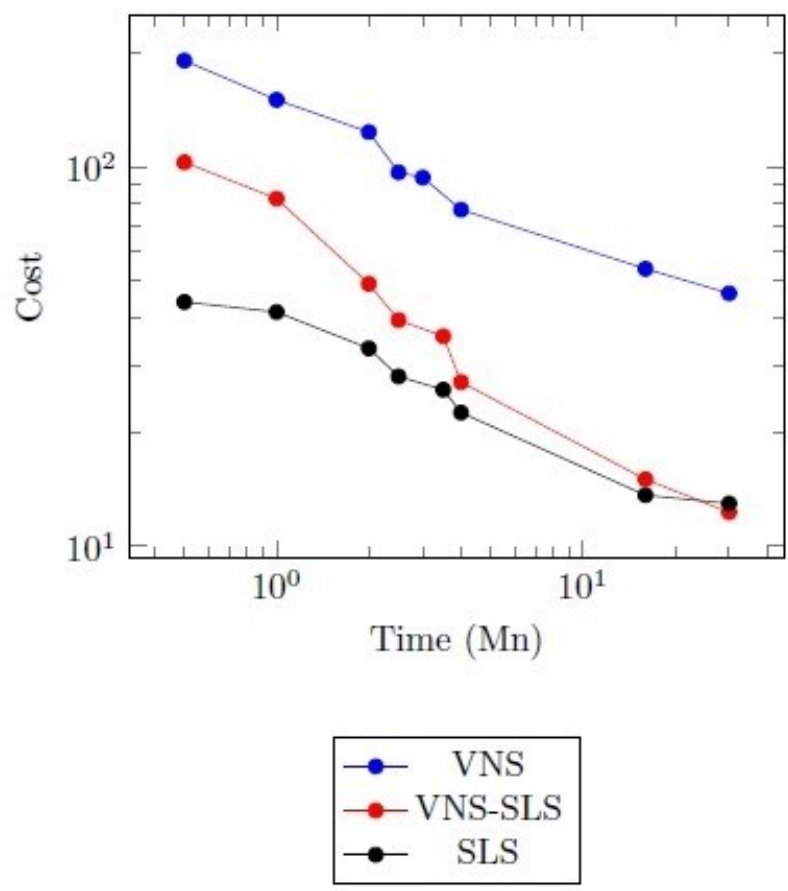

method as a local search in the VNS algorithm, a positive effect and an enhancement is achieved in the quality solution compared to the standard VNS implemented with the local search. Indeed, the SLS technique permits to enhance the neighbor solutions of the VNS search. More precisely, the SLS method permits to ensure a good compromise between intensification and diversification. This helps the method to obtain good results. The diversification phase consists in selecting a random neighbor solution by applying a random move. The intensification phase consists in selecting a neighbor by changing the conflicting node in the solution.

Further, we draw Figure 3 to show the average results obtained for all the considered instances. We can see in Figure 3-a that both SLS and VNS-SLS produced results that are better than VNS for all the Siemens instances. We can see clearly that the combination of SLS and VNS improve the results of VNS particularly for both the instances Siemens 01 and Siemens 03.

For the K instance, we can see in From Figure 3-b that the average solutions obtained by both SLS and VNS-SLS outperform the VNS method. The SLS combine well with the VNS it leads to a good exploration of the search space. It enhances the VNS search process allowing it to get better results.

For the Swisscom and Tiny instances, the behavior of the three methods is almost the same; they all succeed to find the optimal solution.

\subsection{Further Comparison}

Table 3 gives a comparison with a recent work (Lahsinat Y. B., 2015) that used the same formulation and datasets. The work proposed three hyper-heuristics to handle the MI-FAP. We note that the three hyper-heuristics manage the same heuristics but use different manner to select them. The first one namely Hyper(1) is a hyper-heuristic based on a choice function which means that each heuristic has a weight which evaluates its performances. The second namely Hyper(2) uses a random selection of the heuristics and the last one namely Hyper(3) is a hybrid one which uses a probability to decide to use the choice function or a random choice. 
Figure 2. The best results obtained on the Siemens and K instances

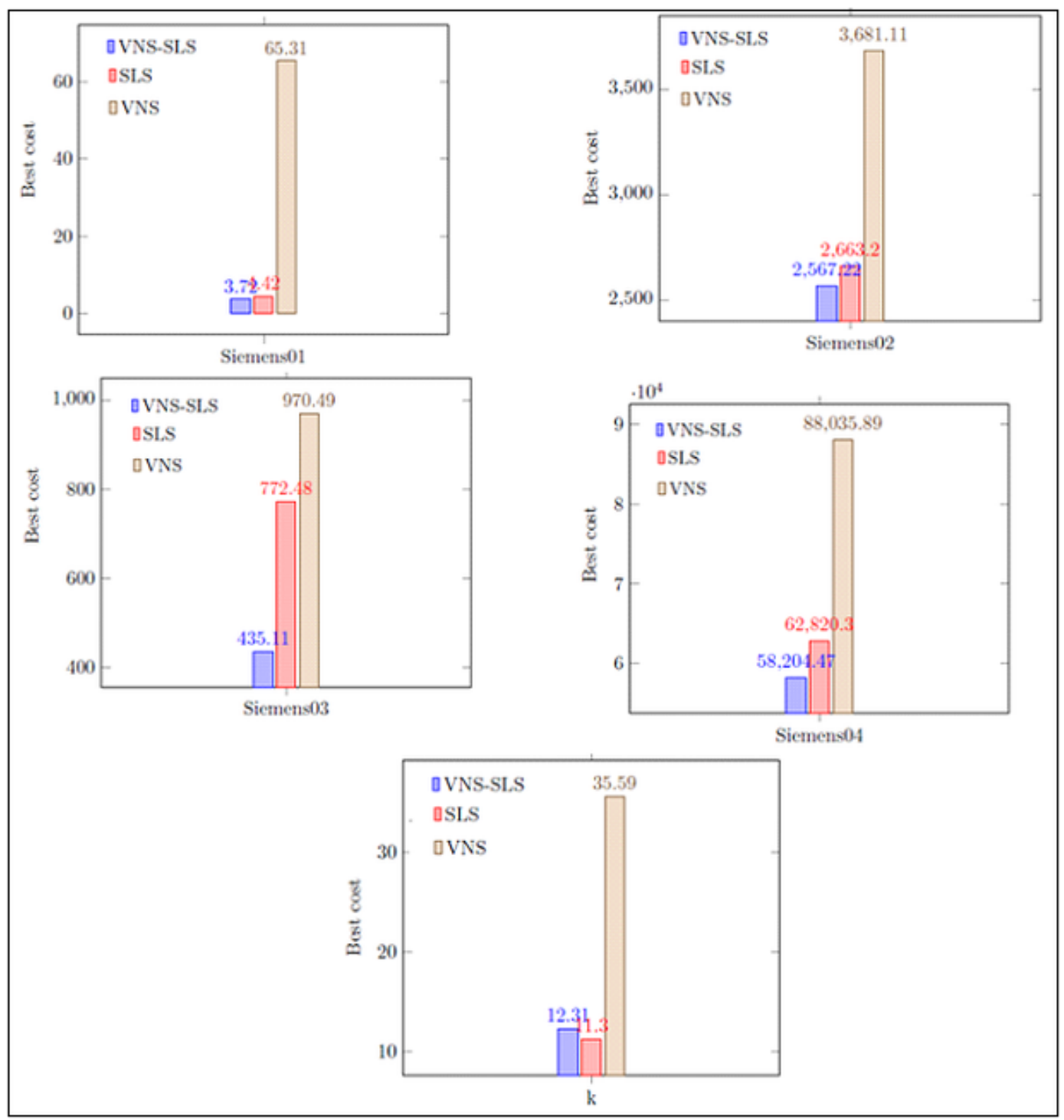

The values reported in Table 3 for the different approaches are the Best and average results recorded from 10 runs for the same overall CPU time equals to 1800 seconds for each run.

We observe that the results presented in Table 3 indicate that our methods outperform those produced by the three hyper-heuristics for all the considered instances. When comparing the results recorded by the VNS-SLS results and the hyper-heuristics, we can observe that for the instances Tiny and Swisscom the methods are comparable.

To show the performance of our methods, we give another comparison in Table 4. We compare our results with those of a Genetic algorithm and a Biogeography-based optimization and two hybrid versions of the Biogeography Based optimization: $\mathrm{HBBO}(1)$ and $\operatorname{HBBO}(2)$ (Lahsinat, 2014). 
Figure 3. The average results obtained on the considered instances

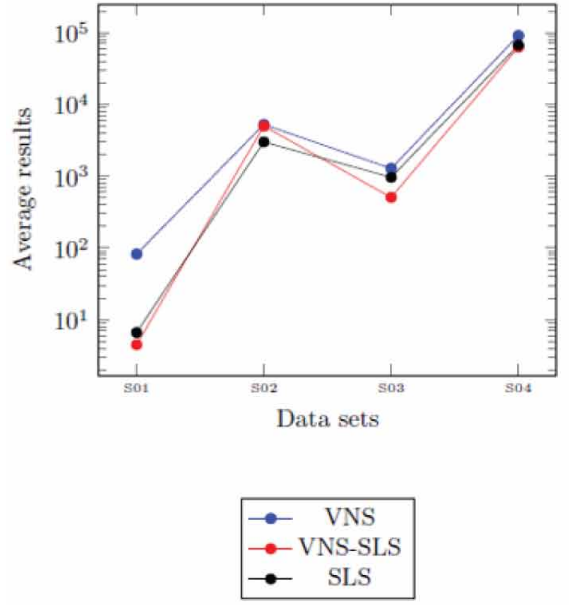

a)- Siemens instances

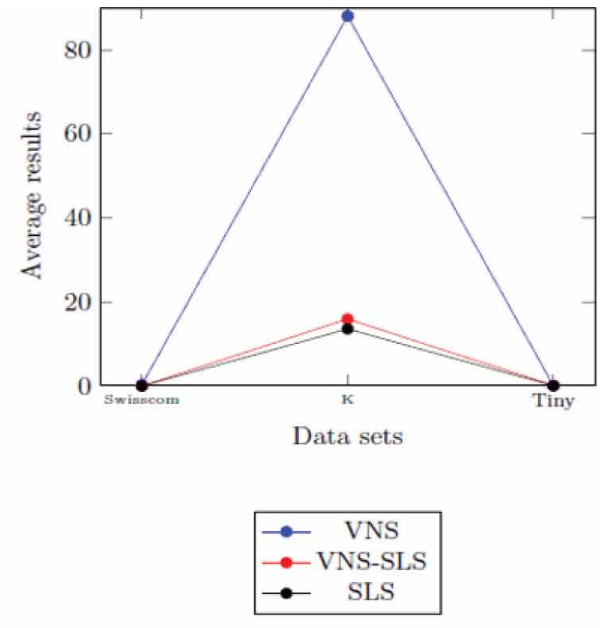

b)- Swisscom, K and Tiny instances

Table 3. A comparison with the hyper-heuristic approaches

\begin{tabular}{|c|c|c|c|c|c|}
\hline \multirow{2}{*}{ Instances } & \multirow{2}{*}{ Results } & \multicolumn{4}{|c|}{ Methods } \\
\hline & & Our Better Achievement & Hyper(1) & Hyper(2) & Hyper(3) \\
\hline Siemens01 & $\begin{array}{l}\text { Best } \\
\text { Average }\end{array}$ & $\begin{array}{l}3.72 \\
4.49 \\
\text { (VNS-SLS) }\end{array}$ & $\begin{array}{l}47.70 \\
88.78\end{array}$ & $\begin{array}{l}21.27 \\
41.60\end{array}$ & $\begin{array}{l}42.79 \\
49.04\end{array}$ \\
\hline Siemens02 & $\begin{array}{l}\text { Best } \\
\text { Average }\end{array}$ & $\begin{array}{l}2567.22 \\
\text { (VNS-SLS) } \\
\underline{3000.81} \text { (SLS) }\end{array}$ & $\begin{array}{l}4990,70 \\
5815,79\end{array}$ & $\begin{array}{l}5187.72 \\
5422.59\end{array}$ & $\begin{array}{l}4199.66 \\
4672.32\end{array}$ \\
\hline Siemens03 & $\begin{array}{l}\text { Best } \\
\text { Average }\end{array}$ & $\begin{array}{l}435.11 \\
506.88 \\
\text { (VNS-SLS) }\end{array}$ & $\begin{array}{l}923,12 \\
1409,34\end{array}$ & $\begin{array}{l}884.64 \\
1004.09\end{array}$ & $\begin{array}{l}702.48 \\
787.99\end{array}$ \\
\hline Siemens04 & $\begin{array}{l}\text { Best } \\
\text { Average }\end{array}$ & $\begin{array}{l}58204.47 \\
62816.43 \\
\text { (VNS-SLS) }\end{array}$ & $\begin{array}{l}108232.4 \\
110959.6\end{array}$ & $\begin{array}{l}76968.78 \\
89047.31\end{array}$ & $\begin{array}{l}87723.83 \\
91096.05\end{array}$ \\
\hline Swisscom & $\begin{array}{l}\text { Best } \\
\text { Average }\end{array}$ & $\begin{array}{l}0 \\
\text { (SLS/VNS-SLS) }\end{array}$ & $\begin{array}{l}0 \\
0\end{array}$ & $\begin{array}{l}0 \\
0\end{array}$ & $\begin{array}{l}0 \\
0\end{array}$ \\
\hline $\mathrm{K}$ & $\begin{array}{l}\text { Best } \\
\text { Average }\end{array}$ & $\begin{array}{l}11.30 \\
13.58 \\
\text { (SLS) }\end{array}$ & $\begin{array}{l}80.22 \\
99.27\end{array}$ & $\begin{array}{l}22.30 \\
43.99\end{array}$ & $\begin{array}{l}100.94 \\
115.29\end{array}$ \\
\hline Tiny & $\begin{array}{l}\text { Best } \\
\text { Average }\end{array}$ & $\begin{array}{l}0 \\
0\end{array}$ & $\begin{array}{l}0 \\
0\end{array}$ & $\begin{array}{l}0 \\
0\end{array}$ & $\begin{array}{l}0 \\
0\end{array}$ \\
\hline
\end{tabular}

It is worth recalling that all the results are given at a CPU time fixed to 1800 seconds. It appears obvious from the results presented in Table 4 that the VNS-SLS and SLS produced better results for all the Siemens and K instances. For the Tiny and Swisscom instances, we can observe that the results are the same. We can claim that SLS combined with the VNS is a good combination. 
Table 4. Comparison with BBO, HBBO(1), HBBO(2) and GA

\begin{tabular}{|c|c|c|c|c|c|c|}
\hline \multirow{2}{*}{ Instances } & \multirow{2}{*}{ Results } & \multirow{2}{*}{ Our better achievement } & \multicolumn{4}{|c|}{ Method } \\
\hline & & & BBO & НBBO(1) & HBBO(2) & GA \\
\hline Siemens01 & $\begin{array}{l}\text { Best } \\
\text { Average }\end{array}$ & $\begin{array}{l}3.72 \\
4.49 \\
\text { (VNS-SLS) }\end{array}$ & $\begin{array}{l}223,62 \\
250,18\end{array}$ & $\begin{array}{l}32.19 \\
44.93\end{array}$ & $\begin{array}{l}100.96 \\
104.40\end{array}$ & $\begin{array}{l}140.68 \\
157.23\end{array}$ \\
\hline Siemens02 & $\begin{array}{l}\text { Best } \\
\text { Average }\end{array}$ & $\begin{array}{l}2567.22 \\
\text { (VNS-SLS) } \\
\underline{3000.81} \text { (SLS) }\end{array}$ & $\begin{array}{l}5941,29 \\
6426,40\end{array}$ & $\begin{array}{l}6325.19 \\
5527.35\end{array}$ & $\begin{array}{l}6325.19 \\
6471.71\end{array}$ & $\begin{array}{l}3778.61 \\
5009.43\end{array}$ \\
\hline Siemens03 & $\begin{array}{l}\text { Best } \\
\text { Average }\end{array}$ & $\begin{array}{l}435.11 \\
\underline{506.88} \\
\text { (VNS-SLS) }\end{array}$ & $\begin{array}{l}1460,65 \\
1544,94\end{array}$ & $\begin{array}{l}1102.76 \\
1202.81\end{array}$ & $\begin{array}{l}1445.11 \\
1498.99\end{array}$ & $\begin{array}{l}1136.92 \\
1276.527\end{array}$ \\
\hline Siemens04 & $\begin{array}{l}\text { Best } \\
\text { Average }\end{array}$ & $\begin{array}{l}58204.47 \\
\underline{62816.43} \\
\text { (VNS-SLS) }\end{array}$ & $\begin{array}{l}108401,08 \\
110830,3\end{array}$ & $\begin{array}{l}97204.81 \\
101117.4\end{array}$ & $\begin{array}{l}110998.65 \\
112104.6\end{array}$ & $\begin{array}{l}101010.6 \\
104288.8\end{array}$ \\
\hline Swisscom & $\begin{array}{l}\text { Best } \\
\text { Average }\end{array}$ & $\begin{array}{l}0 \\
0 \\
\text { (VNS-SLS) }\end{array}$ & $\begin{array}{l}0 \\
0\end{array}$ & $\begin{array}{l}0 \\
0\end{array}$ & $\begin{array}{l}0 \\
0\end{array}$ & $\begin{array}{l}0 \\
0.65\end{array}$ \\
\hline $\mathrm{K}$ & $\begin{array}{l}\text { Best } \\
\text { Average }\end{array}$ & $\begin{array}{l}11.30 \\
13.58 \\
(S L S)\end{array}$ & $\begin{array}{l}98.26 \\
106.27\end{array}$ & $\begin{array}{l}24.30 \\
33.99\end{array}$ & $\begin{array}{l}108.94 \\
118.29\end{array}$ & $\begin{array}{l}32.900 \\
40.788\end{array}$ \\
\hline Tiny & $\begin{array}{l}\text { Best } \\
\text { Average }\end{array}$ & $\begin{array}{l}0 \\
0\end{array}$ & $\begin{array}{l}0 \\
0\end{array}$ & $\begin{array}{l}0 \\
0\end{array}$ & $\begin{array}{l}0 \\
0\end{array}$ & $\begin{array}{l}0 \\
0\end{array}$ \\
\hline
\end{tabular}

The different comparisons provided above shows that the VNS-SLS is better than VNS and SLS stand alone for some instances. Indeed, it improves dramatically the results obtained by VNS on all the instances and improves some other methods published previously. The results obtained by SLS and VNS-SLS on the experimented benchmarks looks to be comparable. The results are too close for some instances. However, the hybrid algorithm VNS-SLS looks stable and robust to deal with great size instances of the MI-FAP. It has a good balance between intensification and diversification and allows a better exploration of the search space.

\section{CONCLUSION}

The frequency assignment is an important task in the design of radio network where the objective is to find an optimal frequency plan that permits to ensure a good quality of service. In this paper, a Variable Neighborhood Search (VNS), a Stochastic Local Search and a new hybrid search method that combines the Variable Neighborhood Search with the Stochastic Local Search are proposed for the MI-FAP. The performances of the proposed algorithms are evaluated and compared using wellknown datasets.

The experiments conducted show the benefits of the SLS as a local Search in the VNS process. The results are very encouraging and the new hybrid method succeeds in finding good results for the considered benchmarks. This hybrid method is still in its cradle and many possibilities and ideas are possible to enhance it and avoid all the hindrances that limit its performance. As a perspective, first it would be interesting to see if we can obtain better results by increasing the search time for the proposed approaches. We also plan to explore other forms of SLS that could be combined with the VNS. Another point that could improve our work is to see if the different steps in the proposed methods could be launched in parallel. 
International Journal of Applied Metaheuristic Computing

Volume $10 \cdot$ Issue $3 \cdot$ July-September 2019

\section{ACKNOWLEDGMENT}

The authors would like to thank the editor and reviewers for careful reading, constructive comments and valuable suggestions that helped us to improve the paper.

Also the authors would like to mention that this work was supported by the PHC Tassili Project -17MDU987. 


\section{REFERENCES}

Aardal, K., van Hoesel, S. P. M., Koster, A. M. C. A., Mannino, C., \& Sassano, A. (2007). Models and solution techniques for frequency assignment problems. Annals of Operations Research, 153(1), 79-129. doi:10.1007/ s10479-007-0178-0

Abunaser, A. M., \& Alshattnawi, S. (2015). Hybridizing artificial bee colony algorithm with multi-parent crossover operator. International Journal of Applied Metaheuristic Computing, 6(2), 18-32. doi:10.4018/ IJAMC.2015040102

Alami, J. E. (2008). Using cultural algorithm for the fixed-spectrum frequency assignment problem. The Journal of Mobile Communication, 2(1), 1-9.

Beckmann, D. K. (1999). Frequency planning with respect to interference minimization in cellular radio networks.

Benmounah, Z. M., Meshoul, S., \& Batouche, M. (2017). Scalable differential evolutionary clustering algorithm for Big Data using map-reduce paradigm. International Journal of Applied Metaheuristic Computing, 8(1), 45-60. doi:10.4018/IJAMC.2017010103

Bezuidenhout, W. (2014). Optimising the frequency assignment problem utilizing particle swarm optimisation [Doctoral dissertation]. University of Johannesburg.

Boucheham, A., \& Batouche, M. (2017). Hybrid wrapper/filter gene selection using an ensemble of classifiers and PSO algorithm. International Journal of Applied Metaheuristic Computing, 8(2), 22-37. doi:10.4018/ IJAMC.2017040102

Boughaci, B. B. (2008). Stochastic Local Search for the Optimal Winner Determination Problem in Combinatorial Auctions. In International Conference on Principles and Practice of Constraint Programming (pp. 593-597). Springer.

Colombo, G. A., \& Allen, S. M. (2010). A comparison of problem decomposition techniques for the FAP. Journal of Heuristics, 16(3), 259-288. doi:10.1007/s10732-009-9116-4

Dorne, R. H. (1999). Tabu search for graph coloring, T-colorings and set T-colorings. In Meta-heuristics (pp. 77-92). Boston, MA: Springer.

Duque-Antón, M., Kunz, D., \& Ruber, B. (1993). Channel assignment for cellular radio using simulated annealing. IEEE Transactions on Vehicular Technology, 42(1), 14-21.

Eisenblatter, A. G. (2002). Frequency planning and ramifications of coloring. In Discussions Mathmatcae Graph Theory (pp. 51-88).

Eisenblätter, A. K. (2000, June 9). COST 259 - Wireless Flexible Personalized Communications. Retrieved from http://fap.zib.de/problems/COST259/

Hale, W. (1980). Frequency assignment: Theory and applications. In Proceedings of the IEEE (pp. 1497-1514).

Hansen, P., \& Mladenović, N. (1997). Variable neighborhood search for the p-median. Location Science, 5(4), 207-226.

Holger, H., \& Hoos, T. S. (2004). Stochastic Local Search: Foundations and Applications. Elsevier.

Kuurne, A. (2001). Mobile Measurement based Frequency Planning in GSM Network. Helsinki University of Technology.

Lahsinat, Y. (2014). Improving Biogeography Based Optimization by Using Stochastic Local Search and Solving MI-FAP Problem in GSM Networks. In Based Optimization (ICSIBO'2014) (pp. 112-119). Mulhouse.

Lahsinat, Y. B. (2015). Trois hyper-heuristiques pour le problème d'affectation de fréquence dans un réseau cellulaire. In 11th Journées Francophones de programmation par contraintes (pp. 184-193). Bordeaux: JFPC.

Lahsinat, Y. B. (2017). Harmony Search Based Algorithms for the Minimum Interference Frequency Assignment Problem. In International Conference on Harmony Search Algorithm (ICHSA2017) (pp. 179-189). Bilbao, Spain: Springer. doi:10.1007/978-981-10-3728-3_18 
International Journal of Applied Metaheuristic Computing

Volume $10 \cdot$ Issue $3 \cdot$ July-September 2019

Lai, X. H., \& Hao, J.-K. (2015). Path relinking for the fixed spectrum frequency assignment problem. Expert Systems with Applications, 42(10), 4755-4767. doi:10.1016/j.eswa.2015.01.025

Leese, R. H. (2002). Methods and Algorithms for Radio Channel Assignment. Oxford: Oxford University Press.

Luna, F. B. (2007). ACO vs EAs for solving a real-world frequency assignment problem in GSM networks. In 9th Annual Conference on Genetic and Evolutionary Computation (pp. 94-101). London: ACM. doi: $10.1145 / 1276958.1276972$

Luna, F. E.-G.-R., Estébanez, C., León, C., Chaves-González, J. M., Nebro, A. J., Aler, R., \& Gómez-Pulido, J. A. et al. (2011). Optimization algorithms for large-scale real-world instances of the frequency assignment problem. Soft Computing, 15(5), 975-990. doi:10.1007/s00500-010-0653-4

Mabed, H., Caminada, A., \& Hao, J. K. (2011). Genetic tabu search for robust fixed channel assignment under dynamic traffic data. Computational Optimization and Applications, 50(3), 483-506.

Metzger, B. H. (1970). Spectrum management technique. In Proceedings of the 38th National ORSA Meeting, Detroit, MI.

Montemanni, R., Moon, J. N., \& Smith, D. H. (2003). An improved tabu search algorithm for the fixed-spectrum frequency-assignment problem. IEEE Transactions on Vehicular Technology, 52(4), 891-901.

Montemanni, R. S., \& Smith, D. H. (2010). Heuristic manipulation, tabu search and frequency assignment. Computers \& Operations Research, 37(3), 543-551. doi:10.1016/j.cor.2008.08.006

Taghavifard, M. T. (2012). Scheduling cellular manufacturing systems using ACO and GA. International Journal of Applied Metaheuristic Computing, 3(1), 48-64. doi:10.4018/jamc.2012010105

Wang, Y., \& Xu, N. (2017). A hybrid particle swarm optimization method for traveling salesman problem. International Journal of Applied Metaheuristic Computing, 8(3), 53-65. doi:10.4018/IJAMC.2017070104

Zemali, E., \& Boukra, A. (2016). Using a bio-inspired algorithm to resolve the multiple sequence alignment problem. International Journal of Applied Metaheuristic Computing, 7(3), 36-55. doi:10.4018/JJAMC.2016070103

Yasmine Lahsinat is a PhD student in computer science. She currently works at the Department of Informatics, University of Science and Technology Houari Boumediene. Yasmine does research on combinatorial optimization, meta-heuristics and Artificial Intelligence. Yasmine is a member of the LRIA Artificial Intelligence Laboratory at the USTHB University.

Dalila Boughaci is a Full Professor in Computer Science at the University of Science and Technology USTHB (Algeria). She got her PhD from the University of Aix-Marseille (France) in 2008 and her "Habilitation" Post-doctoral diploma from USTHB University in 2009. Dalila Boughaci earned a Bachelor of Engineering degree in Computer Science from Algiers University, MS degree in Computer Science and her second PhD in programming systems from the University of Sciences and Technology, Beb-Ezzouar, Algiers in 1997, 2001 and 2008, respectively. Boughaci's current research interests are in the areas of data mining, deep learning, evolutionary computation, artificial intelligence, meta-heuristics, multi-agent systems, network security, credit scoring and e-commerce. She has published several papers on these research topics in journals and conferences and directed several PhD, MS and BS students' projects. Boughaci has taught Parallel computing, machine learning, Web service, Object Oriented Programming, algorithmic, software engineering, databases, Java, Agents and programming languages at Algiers, and served on several program committees. Boughaci is a member of the LRIA Artificial Intelligence Laboratory at the University of Algiers. She is the head of the research team: Optimization, Reasoning and Application of the LRIA Laboratory.

Belaid Benhamou is currently a Research Professor at Aix-Marseille University. He received his mathematical bachelor in 1983, his engineering degree in computer science in 1988 and his PhD in computer science in 1993 at the Aix-Marseille I University. He obtained an Assistant Professor job at the University of Aix-Marseille I in 1995 and in 2001, he obtained his Habilitation thesis after which he got a national qualification for Full University Professor. His research activities are in the field of artificial intelligence: constraint solving, logic and automated theorem proving, logic programming, non-monotonic reasoning, meta-heuristics and combinatorial optimization and data mining. He published over 120 articles and has supervised several doctoral theses on these areas. 